\title{
MULTIDRUG RESISTANT ACINETOBACTER SPP. BLOOD STREAM INFECTION IN A NEONATAL INTENSIVE CARE UNIT OF AN URBAN SPECIALIZED HOSPITAL IN DHAKA
}

\author{
AKTER M ${ }^{1}$, JAHAN N ${ }^{2}$, ISLAM MN ${ }^{3}$, CHOWDHURY F $^{4}$, HOQUE SM $^{5}$, KHANOM S ${ }^{6}$, BEGUM R $^{7}$
}

\begin{abstract}
Background: Acinetobacter spp. are ubiquitous in the environment, that is, soil and water, and occasionally isolated from mucous membrane, secretion, and skin of hospitalized patients, also on surfaces of hospital environment. Antibiotic resistant Acinetobacter spp. blood stream infection is a leading problem. Multidrug resistant Acinetobacter spp. blood infection in the neonatal intensive care unit (NICU) patients create a great problem in hospital settings.

Methods: This study was carried out from January to December 2011 based on the clinical specimens obtained from suspected cases of septicemia patients admitted in the NICU of Addin Women's Medical College Hospital, Dhaka, Bangladesh. Blood collected from suspected cases by maintaining strict aseptic precaution and sent immediately to microbiology laboratory of the same institution. Samples were also taken from different sites in NICU and Department of Obstetrics \& Gynaecology operation theatre for detection of the sources of infection in outbreak situation. Isolated organisms were identified by standard bacteriological method and drug susceptibility test had been done by modified Kirby Bauer disk diffusion technique.

Results: A total 87 blood culture positive cases were included in this study. Out of 87 culture positive cases Acinetobacter spp. was the predominant pathogen 32 (35.58\%). Acinetobacterspp isolated from the blood of newborn and the mortality rate was 3.33\%. Acinetobacter blood stream infection is more common in the first 7 days of life (early onset), birth weight less than 2 $\mathrm{kg}$ (56.6\%), also affected the baby with birth weight more than $2.5 \mathrm{Kg}(23.3 \%)$ and most of the neonates having gestational age less than 37 weeks (70\%). Twenty four babies (80\%) were delivered in the same hospital. Acinetobacter spp. showed increased resistance against majority of antibiotics such ascolistin 3.12\%, levofloxacin 40.62\%, imipenem 53.12\%, cotrimeoxazole $54.54 \%$, pipericillin tazobactum 59.37\%, amikacin 75\%, ciprofloxacin $78.12 \%$,gentamycin $84.37 \%$, amoxyclavulonic acid 93.75\%, ceftriaxone, ceftazidime and cefixime $96.87 \%$ cefuroxime, cephradineand ampicillin 100\% respectively. We categorized the Acinetobacter spp. in to 3 categories, such as multidrug resistant (MDR) 37.5\%, extensive drug resistant (XDR) $28.12 \%$ and pandrug resistant (PDR) 3.12\% Miscellaneous pattern of sensitivity $31.15 \%$. A cluster of cases occurred in month June, which was quite higher than other months, Surveillance had been done. Acinetobacter spp. isolated from the environment of operation theatre of Obstetrics and Gynea department. In NICU, Acinetobacter spp were isolated from suction water, suction tube, incubator door handle and body surface of the neonates. Acinetobacter spp. which were isolated from suction tube and suction water were only sensitive to colistin and resistant to other drugs. Two Acinetobacter spp. were alsoisolated from OT environment and body surface of neonates which were found sensitive to only imipenem and colistin. Acinetobacter spp. isolated from incubator door handle was sensitive to colistin, imipenem, amikacin, gentamycin, levofloxacin, ciprofloxacin, piperacillin tazobactum and cotrimoxazole.
\end{abstract}

Key words: Multidrug resistance, Acinetobacter, septicemia, antibiotic resistance, nosocomial infection.

J Dhaka Med Coll. 2015; 24(1) : 47-52.

1. Dr. Mursheda Akter, Professor and Head, Department of Microbiology, Dhaka Central International Medical College, Dhaka.

2. Dr. Nasim Jahan, Associate Professor, Department of Neonatology, Ad-din Women's Medical College, Dhaka.

3. Dr. Md. Nazrul Islam, Associate Professor, Department of Nephrology, Dhaka Medical College, Dhaka.

4. Dr. Ferdousi Chowdhury, Associate Professor, Department of Obstetrics \& Gynaecology, Ad-din Women's Medical College, Dhaka.

5. Dr. Syada Monira Hoque, Assistant Professor, Department of Microbiology, Dhaka Central International Medical College, Dhaka.

6. Dr. Sayeda Khanom, Assistant Professor, Department of Pharmacology, Dhaka Central International Medical College, Dhaka.

7. Dr. Rahana Begum, Assistant Professor, Department of Community Medicine, Dhaka Central International Medical College, Dhaka.

Correspondence: Dr. Mursheda Akter, Professor and Head, Department of Microbiology, Dhaka Central International Medical College, Dhaka. 


\section{Introduction}

Acinetobacter baumannii is an opportunistic pathogen that is frequently involved in out breaks of infection occurring mostly in intensive care units from contaminated common sources, e.g. ventilator equipments, catheters, etc ${ }^{1}$. Acinetobacter spp. areaerobic Gram negative, non-motile, non-spore forming encapsulated or biofilm producing coccobacilli belonging to family Neisseriaceae ${ }^{2}$.Commonly present in environment (water, soil) and hospital (catheters, lotions, ventilator equipments). Acinetobacter baumannii is the major species of Acinetobacter. It is an important pathogen found to be associated with wide spectrum of nosocomial infection. Risk factors for Acinetobacter infection are hospitalization, ICU, surgery, antibiotic exposure and catheters $^{3}$. It is clearly pathogenic when recovered from blood and normally sterile body sites $^{3}$. Septicemia is a major cause of morbidity and mortality in newborns, especially in developing countries. There are many studies documented worldwide in the literatures emphasizing the Acinetobacter spp. as an important nosocomial agent of septicemia in neonatal intensive care units ${ }^{4-6}$. Management of multidrug resistant Acinetobacter spp. infections is a great challenge for physicians and clinical microbiologist. Acinetobacter spp. has the ability to survive in a hospital milieu and its ability to persist for extended periods of time on surfaces makes it a frequent cause for health care associated infection and it leads to multiple outbreaks ${ }^{2}$. Early diagnosis and appropriate antimicrobial therapy of septicemia are of utmost importance to prevent morbidity and mortality.

Definitions of multidrug-resistant Acinetobacter species vary when referring to a wide array of genotypes and phenotypes ${ }^{7}$. Different terms like 'multidrug resistant (MDR)', 'extensive drug resistant (XDR),' and 'pandrug resistant (PDR)' have been used with varied definitions to describe the extent of antimicrobial resistance among Acinetobacter spp. However, to date, unlike Mycobacterium tuberculosis, internationally, there are no accepted definitions for the extent of resistance in the bacteria. Arbitrarily used terms have thus caused great confusion making it difficult for the available literature to be analyzed ${ }^{8}$.

In the present study 'MDR Acinetobacter spp.' would be defined as the isolate resistant to at least three classes of antimicrobial agents such as all penicillins and cephalosporins (including inhibitor combinations), fluroquinolones, and aminoglycosides. 'XDR Acinetobacter spp.'would be the Acinetobacter spp. isolate that is resistant to the three classes of antimicrobials described above (MDR) and shall also be resistant to carbapenems. Finally, 'PDR Acinetobacter spp.' would be the XDR Acinetobacter spp. that is resistant to polymyxins and tigecycline. These definitions further help to clearly define the extent of resistance and rational antimicrobial therapy ${ }^{9}$.

The current study highlighted Acinetobacter spp. as important pathogens in neonatal blood stream infection, relationship of Acinetobacter infection with onset of disease, gestational age, birth weight, inborn or out born delivery, identification of the sources of infection in outbreak situation and evaluation of its antimicrobial sensitivity.

\section{Methods}

The study was done in Ad-din Women's Medical College and Hospital, Dhaka, Bangladesh, from January to December of 2011. Blood collected from suspected cases by maintaining strict aseptic precaution in to blood culture bottle and sent immediately to microbiology laboratory of the same hospital. Only symptomatic babies who have had blood culture positive for Acinetobacter spp. were included in the study. Blood culture test was done by FAN method. Subcultures of the positive samples were done on blood agar and McConkey's agar media. Organisms were identified by standard bacteriological method and drug susceptibility test was done by modified Kirby Bauer disk diffusion technique ${ }^{10-12}$. The surveillance study to detect the sources of infection was carried out. Samples of NICU from various articles were taken for cultures. Swabs were taken from suction tubes, water for suction, incubator door handle, baby cots, body surface of the newborn and hand swabs of nurses working in NICU. Samples from labor room and Obstetrics 
\& Gynaecology operation theatre were also taken. wabs were taken from baby trays, resuscitation equipments, suction apparatus. Air sampling was done by keeping two open plates of blood agar and McConkey's agar in to the operation rooms and NICU for 30 minutes ${ }^{13,14}$. All the samples and plates were immediately sent to microbiology laboratory and samples were immediately inoculated in to blood agar and MacConkey's agar media and plates were incubated in to incubator at $37^{\circ} \mathrm{C}$. Isolated organisms were identified by standard biochemical test. Identification of Acinetobacter spp. was made as per standard criteria ${ }^{11}$. Susceptibility pattern were done by modified Kirby Bauer disc diffusion technique ${ }^{10}$.

\section{Results}

Table-I shows that organisms isolated from the blood of the newborn are Acinetobacter spp. 32(34.48\%), followed by Pseudomonas spp. 19(21.83\%), CoNS 16(18.39\%), Klebsiella and Moraxella spp.6(6.89\%) respectively, Staph. aureus 4(5.97\%) Enterobacter spp.3(3.44\%), Esch. coli (2.29\%) and Enterococci spp. $1(1.49 \%)$.The other results are shown in the tables II-V.

\section{Table-I}

Different organisms isolated from blood of neonates in NICU

\begin{tabular}{lcc}
\hline Name of organisms & Number & Percentage (\%) \\
\hline Acinetobacter spp. & 32 & 34.48 \\
Pseudomonas spp. & 19 & 21.83 \\
CoNS & 16 & 18.39 \\
Klebsiella spp. & 06 & 6.89 \\
Moraxella spp. & 06 & 6.89 \\
Staph. aureus & 04 & 5.97 \\
Enterobacter spp. & 03 & 3.44 \\
Esch.coli & 02 & 2.29 \\
Enterococci spp. & 01 & 1.49 \\
\hline Total & 87 &
\end{tabular}

Table-II

Relationship of Acinetobacter spp.blood infectionwith onset of disease mortality rate, birth weight, place of deliveryand gestational age $(n=30)$

\begin{tabular}{|c|c|c|c|c|c|c|c|c|c|c|}
\hline \multirow[t]{2}{*}{ Mortality } & \multicolumn{2}{|c|}{$\begin{array}{l}\text { Age of } \\
\text { onset }\end{array}$} & \multicolumn{3}{|c|}{$\begin{array}{l}\text { Birth weight } \\
\qquad(\mathrm{kg})\end{array}$} & \multicolumn{2}{|c|}{$\begin{array}{c}\text { Gestational } \\
\text { age (week) }\end{array}$} & \multicolumn{2}{|c|}{$\begin{array}{l}\text { Place of } \\
\text { delivery }\end{array}$} & \multirow[t]{2}{*}{ Total } \\
\hline & Early & Late & $1-1.9 \mathrm{~kg}$ & $2-2.5 \mathrm{~kg}$ & $>2.5 \mathrm{~kg}$ & $<37$ wks & $>37$ wks & In-born & Out- born & \\
\hline 1 & 21 & 09 & 17 & 06 & 07 & 21 & 9 & 24 & 6 & \\
\hline (3.33\%) & $(70 \%)$ & $(30 \%)$ & (56.6\%) & $(20 \%)$ & $(23.3 \%)$ & $(70 \%)$ & $(30 \%)$ & $(80 \%)$ & $(20 \%)$ & 30 \\
\hline
\end{tabular}

Table-III

Susceptibility pattern of Acinetobacter spp. to different antimicrobial agents $(n=32)$

\begin{tabular}{|c|c|c|c|c|c|c|}
\hline \multirow[t]{2}{*}{ Antibiotics } & \multicolumn{2}{|c|}{$\begin{array}{l}\text { Sensitive } \\
\text { Resistant }\end{array}$} & \multicolumn{2}{|c|}{$\begin{array}{c}\text { Intermediate sensitive } \\
\text { Resistant }\end{array}$} & \multicolumn{2}{|c|}{ Moderate } \\
\hline & No. & $\%$ & No. & $\%$ & No. & $\%$ \\
\hline Colistin & 31 & 96.87 & & & 01 & 3.12 \\
\hline Imepenem & 15 & 46.87 & & & 17 & 53.12 \\
\hline Piperacillin-tazobactum & 13 & 40.62 & & & 19 & 59.37 \\
\hline Amikacin & 08 & 25 & & & 24 & 75 \\
\hline Levofloxacin & 08 & 25 & 11 & 34.37 & 13 & 40.62 \\
\hline Ciprofloxacin & 06 & 18.75 & 01 & 3.12 & 25 & 78.12 \\
\hline Gentamycin & 05 & 15.62 & & & 27 & 84.37 \\
\hline Ceftriaxone & 01 & 3.12 & & & 31 & 96.87 \\
\hline Ceftazidime & 01 & 3.12 & & & 31 & 96.87 \\
\hline Cefuroxime & 01 & 3.12 & & & 31 & 96.87 \\
\hline Cephradin & 00 & 00 & & & 32 & 100 \\
\hline Ampicillin & 00 & 00 & & & 32 & 100 \\
\hline Amoxyclavulonic acid & 02 & 6.25 & & & 30 & 93.75 \\
\hline Cotrimoxazole & 15 & 46.87 & & & 17 & 53.12 \\
\hline
\end{tabular}


Table-IV

Rate of MDR, XDR and PDR Acinetobacter spp. (n=32)

\begin{tabular}{llcc}
\hline Categories of Acinetobacter & Definition & No. & Percentage \\
\hline MDR & Resistant to & 12 & $37.5 \%$ \\
& 1. All cephalosporins and inhibitor combination & & \\
& 2. Fluroquinolones, & & \\
& 3. Aminoglycosides. & 09 & $28.12 \%$ \\
XDR & MDR+ Resistant to carbapenems & 01 & $3.12 \%$ \\
PDR & XDR + Resistant to polymixin E(colistin) & 10 & $31.25 \%$ \\
Unclassified & Miscellaneous pattern of sensitivity & 10 & \\
\hline
\end{tabular}

Table-V

Acinetobacter spp. isolated from different article in NICU, Obstetrics \& Gynaecology OT (OBG-OT)

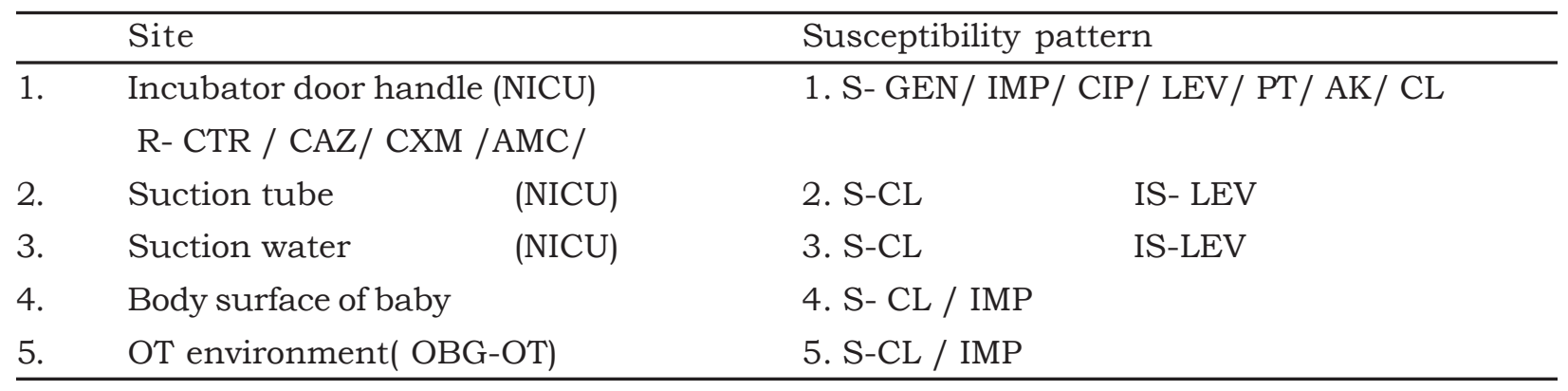

Note: S(Sensitive), IS- Intermediate Sensitive, NICU(Neonatal ICU), GEN (gentamycin), IMP(imipenem), CIP (ciprofloxacin), LEV ( levofloxacin), PT( piperacillin-tazobactum), AK (amikacin), CL (colistin).

\section{Discussion}

Acinetobacter spp. has emerged as an important pathogen causing life threatening infections both in community and hospital. Rapid emergence of multidrug-resistant Acinetobacter spp.has further made the situation $\mathrm{critical}^{2}$. Multidrug resistant noscomialAcinetobacter spp.septicemia may cause severe clinical diseases in neonate that is associated with a high mortality ${ }^{15}$. From January to December 2011 eighty seven organisms were isolated from the blood of neonates. Out of 87 blood culture positive cases Acinetobacter spp.was the most prevalent organism in NICU patients (34.48\%). Isolation rate of Acinetobacter spp. from blood samples of neonates in various study varies from $8.3 \%$ to $15.2 \%^{6,16}$.

NICU of Ad-din women's Medical Collage Hospital, Dhaka, is a referral centre for babies born outside of this hospital. In the present study, we found that Acinetobacter spp. septicemia was more common for the babies in the first 7 days of life (early onset) having birth weight less than $2 \mathrm{~kg}$, baby took born before 37 weeks of pregnancy. Among twenty four neonates having Acinetobactersepticemia, 20 of them delivered in the same hospital by the method of LUCS and four by NVD. Acinetobacter also affected he average gestational age (30\%) and average birth weight babies(23.3\%). Acinetobacter spp. blood infection can even occur in the babies without being under intensive care or antibiotics, mechanical ventilation or having indwelling catheters 17. Institutional birth, preterm birth, and low birth weight were identified the most frequent risk factors $6,16,17$. This might be because of prevalence of multi-drug resistant strains in the hospital environment ${ }^{6}$. Mortality rate is lowered (3.33\%) in our study compare with others ${ }^{18}$. It might be due to involvement of babies more than higher birth weight and without prior sever illnessv. 
Acinetobacter spp. has been implicated in many outbreaks in the neonatal sepsis in NICUt 2 13. From January to May of 2011,15 Acinetobacter spp. isolated from blood of suspected cases of septicemia patients in Neonatal intensive care(NICU) in Ad-din women's medical college hospital, but in the month of June 2011, 12 Acinetobacter spp. were isolated from blood of neonates, rate was surprisingly more than other months, so we took it as an outbreak, and nine of them are XDR (MDR + resistance to carbepenam).

Surveillance study was done and samples were taken from several sites in NICU and operation theatre of OBG department. Acinetobacter spp. was isolated from incubator door handle, suction tube, suction water and body surface of the baby in NICU and it was also isolated from OT environment during the outbreak time. Organisms isolated from suction water and suction tube are XDR, these susceptibility pattern correlates with the susceptibility pattern of organisms isolated from the patients at the time of outbreak. This time some of the sucker machines in NICU were unserviceable and a single sucker machine being used for several babies. Enforcement of hospital infection prevention control measure had been done, in the following six months only five Acinetobacter $s p$. were isolated from the blood of newborn.

In the current study isolated Acinetobacter spp.showed high level of resistanceto most of the antibiotic compared with other studies done in different places ${ }^{16,17}$.In present study more than $96 \%$ of isolates were resistant to $3^{\text {rd }}$ generation cephalosporin, followed by gentamycin $84.37 \%$, ciproflaxacin $78.12 \%$, amikacin $75 \%$, piperacillin-tazobactum $59.37 \%$, imipenem and cotrimoxazole $53.12 \%$, levofloxacin $40.62 \%$ and colistin $3.12 \%$. Ampicillin and $1^{\text {st }}$ and $2^{\text {nd }}$ generation cephalosporin found $100 \%$ resistant. In a surveillance study of the antibiotic susceptibility pattern of the ICUs of five Europian countries had done and the prevalence of resistance of Acinetobacter spp. to gentamycin was $0-81 \%$, amikacin 10-51\%, ciprofloxacin 19 $81 \%$, ceftazidime $0-81 \%$, piperacillin-tazobactum 36-75\% and imipenem 5-19\% ${ }^{19}$. In present study
$38 \%$ of Acinetobacter spp. were MDR, 28.12\% XDR, 3.12\% PDR and miscellaneous pattern of sensitivity $31.25 \%$.A. baumannii was isolated most in a study were multidrug resistant $(M D R)^{20}$. According to Abbo et al., 2005, MDR criteria were defined when resistant to all of studied antibiotics, that is, piperacillintazobactam, cefepime, ceftazidime, aztreonam, ciprofloxacin, gentamicin, and tobramycin, but could be sensitive to amikacin, ampicillinsulbactam, imipenem, meropenem, and minocycline ${ }^{21}$. Several reports showed that the usage of broad spectrum antibiotics affected normal flora and induced MDR A. baumannii ${ }^{22,23}$. Acinetobacter spp. are rapidly spreading with emergence of extended resistance to even newer antimicrobials. They have the ability to acquire resistance at a much faster than other gram-negative organism ${ }^{9}$. Due to their ease of survival in the hospital environment, they have immense potential to cause nosocomial outbreaks. In addition to antibiotic resistance, their biofilm forming ability plays a crucial role in their in-vitro and in-vivo survival ${ }^{9}$.

\section{Conclusion}

In the present study, we concluded that Acinetobacter spp. is one of the major pathogens in NICU, can reach in outbreak proportions and may involve babies with normal birth weight. Most of the Acinetobacter spp. are multidrug resistant. Colistin is the drug of choice for blood stream infection of ICU patient caused by Acinetobacter spp. To decrease the spread of Acinetobacter infections and reduce the pace of emergence of resistance in MDR Acinetobacter, it is important to promote the rational use of antimicrobials, with implementation and monitoring of the Antibiotics Stewardship Program in hospitals. Hospital infection prevention committee can play a key role to prevent nosocomial infection.

\section{References}

1. Paterson DL. The epidemiological profile of infections with multidrug-resistant Pseudomonas aeruginosa and Acinetobacter species. ClinInfec Dis 2006; 43: S43-8.

2. Bergonge-Berezin E, Towner JK. Acinetobacter spp. as nosocomial pathogens: Microbiological, clinical 
and epidemiological features. Clin Microbiol Rev 1996; 9: 148-51.

3. Bartlett, JG. Acinetobacter baumannii: Johns Hopkins Medicine POC-IT Guides. Last updated: November $\quad 15, \quad 2011 \mathrm{http}$ // www.hopkinsguide.com/hopkins/ud/view / Johns_Hopkins_ABX_Guide/54003/all/A.

4. Touati A, Achour W, Cherif A, et al.Out break of Acinetobacter baumannii in neonatal intensive care unit: antimicrobial susceptibility and genotyping analysis. Ann Epidemiol 2009; 19:372-8.

5. Jarousha AA, El Qouqa IA, Jadba AE, Al Afifi AS. Acinetobacter baumanniiinfection in the neonatal intensive care unit. Iranian J Health 2008: 37:107-12.

6. Arora U, Jaitwani J. Acinetobacterspp.:an emerging pathogen in neonatal septicaemia in Amritsar. Indian J Med Microbiol 2006;24:81.

7. Falagas ME, Koletsi PK, Bliziotis IA. The diversity of definitions of multidrug-resistant (MDR) and pandrug-resistant (PDR) Acinetobacter baumannii and Pseudomonas aeruginosa. J Med Microbiol2006;55: 1619-29.

8. Falagas ME, Karageorgopoulos DE. Pandrug resistance (PDR), extensive drug resistance (XDR), and multidrug resistance (MDR) among Gramnegative bacilli: need for international harmonization in terminology. Clin Infect Dis2008;46: 1121-2.

9. Manchanda V, Sanchaita S, Singh NP. Multidrug resistant Acinetobacter. J Global Infect Dis 2010; 2(3): 291-304.

10. Kirby AW, Sherris JC, Truck M. Antibiotic susceptibility testing by a standardized single disk method. AmJClinPathol1996; 36(3): 493-6.

11. Collee JG. Laboratorystrategy in thediagnosis of infective syndrome. In: Duguid JP, Fraser AG, Marimon BP. eds.Mackie and McCartney Practical Medical Microbiology. $14^{\text {th }}$ ed. New York: Churchill Livingstone; 1996.

12. Chessbrough M. Medicallaboratory manual for tropical countries. Vol.II. Cambridge: ELBS; 1984.

13. Von Dolinger de Brito D, Oliveira EJ,Abdullah VO, et al. An outbreak of Acinetobacter baumannii septicemia in a neonatal intensive care unit of a university hospital in Brazil. Brazil J Infect Dis 2005; 9:301-9.

14. Mohiuddin M, Haq A,Haq M, Huq F.Microbilogy of nosocomial infection in tertiary hospital of Dhaka city and its impact. Bangladesh J Med Microbiol 2010; 4: 32-7.

15. Cisneros JM, Reyes MJ, Pachon J, et al. Bacteremia due to Acinetobacter baumannii: epidemiology, clinical findings and prognostic features. Clin Infect Dis 1996;22:1026-32.

16. Shete VB, Ghadage DP, Muley VA, Bhore AV. Acinetobacter septicemia in neonate admitted to intensive care unit. J Lab Phys 2009;1: 73-6.

17. Ashit M, Shudhir M, Geetha J, et al. Acinetobacter Sepsis in Newborns. Indian Pediatr1998;35:2732 .

18. Seifert H, Strate A, Pulverer G. Nosocomial bacteremia due to Acinetobacter baumannii: clinical features, epidemiology and predictors of mortality. Medicine 1995; 74: 340-9.

19. Hanberger H, Garcia-Rodriguez JA, Gobernado M, et al. Antibiotic susceptibility among aerobic gramnegative bacilli in intensive care units in 5 European countries. French and Portuguese ICU Study Groups. JAMA 1999;281: 67-71.

20. Tjoa E, Moehario LH, Rukmana A, Rohsiswarrno R. Acinetobacter baumannii: role in blood stream infection in neonatal unit, Dr. CiptoMangunkusumo Hospital, Jakarta, Indonesia.Int J Microbiol 2013;

21. A Abbo S, Navon-VeneziaO, Hammer-MuntzT, et al. Multidrug-resistant Acinetobacter baumannii. Emerg Infect Dis 2005; 11(1): 22-9.

22. Agustí CM, Pujol MJ,ArgerichMJ, et al. Short-term effect of the application of selective decontamination of the digestive tract on different body site reservoir ICU patients colonized by multiresistant Acinetobacter baumannii. J AntimicrobChemother 2002; 49: 205-8.

23. Camp, Tatum OL. A review of Acinetobacter baumannii as a highly successful pathogen in times of war. Lab Med 2010; 41: 649-57. 